\title{
Sums of Alternating Matrices and Invertible Matrices
}

\author{
T. Y. Lam and R. G. Swan
}

\begin{abstract}
A square matrix is said to be alternating-clean if it is the sum of an alternating matrix and an invertible matrix. In this paper, we determine all alternating-clean matrices over any division ring $K$. If $K$ is not commutative, all matrices are alternating-clean, with the exception of the $1 \times 1$ zero matrix. If $K$ is commutative, all matrices are alternating-clean, with the exception of odd-size alternating matrices, and six special $2 \times 2$ matrices in the case where $K$ is $\mathbb{F}_{2}$, the field of two elements. Similar results are obtained over semilocal rings.
\end{abstract}

\section{Introduction}

One of the earliest results in the theory of additive decompositions of linear transformations (or matrices) was that of D. Zelinsky [Ze], who proved that, with a trivial exception over the field of two elements, every linear endomorphism of a (possibly infinite-dimensional) vector space over a division ring can be written as the sum of two nonsingular transformations. The problem of decomposing linear transformations into a sum of idempotents was considered by K. G. Wolfson [Wo]. More recently, Searcóid [Se], Nicholson, Varadarajan and Zhou [NVZ] showed that every linear transformation of a (possibly infinite-dimensional) vector space over a division ring is the sum of an idempotent transformation and an invertible transformation; for a generalization of this to continuous modules, see [CK].

In this paper, we consider the problem of decomposing a square matrix into the sum of an alternating matrix and an invertible matrix. The motivation for this work came from our investigation of a class of sequences, called sectionable sequences, over a commutative ring $K$. (By definition, a sequence $a_{1}, \ldots, a_{n}$ over $K$ is said to be sectionable if there exists another sequence $b_{1}, \ldots, b_{n}$ such that $\sum a_{i} b_{i}=0$ and $\left.\sum a_{i} K=\sum b_{i} K.\right)$ In this study, the idea of decomposing a matrix into the sum of an alternating matrix and an invertible matrix comes to the fore; see [LS]. While the study of such decompositions in [LS] is carried out (and applied) in a ring-theoretic setting, the classical case of fields (and division rings) did not seem to have been treated before in the literature. In this note, we focus our study on this classical case, and prove the following two results.

1991 Mathematics Subject Classification. Primary 15A33, 15A57; Secondary 16L30, 16K99.

Key words and phrases. Linear algebra, additive decompositions, alternating matrices, invertible matrices, fields, division rings, simple artinian rings, semilocal rings, alternating-clean. 
Theorem A. Over a noncommutative division ring $K$, every matrix $M \in \mathbb{M}_{n}(K)$ is the sum of an alternating matrix and an invertible matrix, with the single exception of the $1 \times 1$ matrix $M=(0)$.

Theorem B. Over any field $K$, every matrix in $\mathbb{M}_{n}(K)$ is the sum of an alternating matrix and an invertible matrix, with the exception of all odd-size alternating matrices (over all $K$ ), and the following six matrices

$$
\left(\begin{array}{ll}
0 & 1 \\
0 & 0
\end{array}\right),\left(\begin{array}{ll}
1 & 1 \\
0 & 0
\end{array}\right),\left(\begin{array}{ll}
0 & 1 \\
0 & 1
\end{array}\right),\left(\begin{array}{ll}
0 & 0 \\
1 & 0
\end{array}\right),\left(\begin{array}{ll}
1 & 0 \\
1 & 0
\end{array}\right) \text {, and }\left(\begin{array}{ll}
0 & 0 \\
1 & 1
\end{array}\right)
$$

over $\mathbb{F}_{2}$, the field of two elements.

Note that the last three matrices here are the transposes of the first three. Together, the six matrices in (1.1) can be characterized as those $M=\left(m_{i j}\right) \in$ $\mathbb{M}_{2}\left(\mathbb{F}_{2}\right)$ with the property that $m_{11} m_{22}=0 \neq m_{12}-m_{21}$. The remaining ten matrices in $\mathbb{M}_{2}\left(\mathbb{F}_{2}\right)$ are those in $\mathrm{GL}_{2}\left(\mathbb{F}_{2}\right) \cong S_{3}$, and the four matrices $0, E_{11}, E_{22}$, and $E_{11}+E_{12}+E_{21}+E_{22}$ (each of which is the sum of the alternating matrix $E_{12}-E_{21}$ and a matrix in $\left.\mathrm{GL}_{2}\left(\mathbb{F}_{2}\right)\right)$. Throughout this paper, $E_{i j}$ denote the matrix units, and $\mathrm{GL}_{n}(K)$ denotes the $n \times n$ general linear group over a (possibly noncommutative) ring $K$.

The proof of Theorem A and Theorem B, given in $\S 3$ below, is essentially constructive in the sense that, if a matrix $M$ over a division ring $K$ is not one that is excluded by Theorem A or Theorem B, the proof will lead to an explicit construction of a representation of $M$ as a sum of an alternating matrix and an invertible matrix.

In $\S \S 5-6$ of this paper, we extend Theorems A and B to commutative (and "most" noncommutative) semilocal rings. The relevant results here are Theorem C in $\S 5$, and (6.1), (6.2). Over a general commutative ring, however, the determination of matrices $D$ that are sums of an alternating matrix and an invertible matrix is harder and much more subtle. This case will be considered in some detail in a sequel to this paper.

\section{Alternating-Clean Matrices over Rings}

In a purely ring-theoretic context, W. K. Nicholson [Ni] studied elements in rings that can be decomposed into a sum of an idempotent element and an invertible element. He called such ring elements clean. If every element in a ring $S$ is clean, Nicholson showed that $S$ is an exchange ring (in the sense of Warfield).

A more general notion of "cleanness" may be defined as follows. Let $\mathcal{A}$ be a set of elements in a ring $S$. An element $s \in S$ is said to be $\mathcal{A}$-clean if $s$ can be written as a sum of an element in $\mathcal{A}$ and an element in $\mathrm{U}(S)$ (the group of units in $S$ ); in other words, up to a perturbation by an element of $\mathcal{A}, s$ "becomes" a unit in $S$. In [Ni], $\mathcal{A}$ is taken to be the set of idempotent elements in $S$. In this case, we get the clean elements of $S$ as defined in the last paragraph. Nicholson's notion of cleanness has proved to be a useful paradigm in ring theory, and has been extended in various ways by replacing the set of idempotents in $S$ by different choices of the set $\mathcal{A} \subseteq S$; e.g. the set of units, nilpotent elements, or von Neumann regular elements in $S$. 
Following Albert [Al], we call a square matrix $A$ alternate (or more popularly, alternating) if $A$ is skew-symmetric and has zero elements on the diagonal. In this note, we investigate the $\mathcal{A}$-clean notion in a matrix ring $S=\mathbb{M}_{n}(K)$ by taking $\mathcal{A}$ to be the $(K, K)$-bimodule of alternating matrices $\mathbb{A}_{n}(K) \subseteq S$. Since our interest in this paper is not only confined to the case of division rings, we give the following definition for a general ring $K$.

Definition 2.1. A matrix $M \in \mathbb{M}_{n}(K)$ is said to be alternating-clean (or $\mathbb{A}$ clean for short) if $M$ can be expressed as a sum $A+U$, where $A \in \mathbb{A}_{n}(K)$ and $U \in \mathrm{GL}_{n}(K)$.

The following are some basic observations on $\mathbb{A}$-clean matrices that will be used freely in the rest of this paper.

Remarks 2.2. (1) Neeedless to say, invertible $n \times n$ matrices are $\mathbb{A}$-clean. For $n=1$, the converse also holds: a $1 \times 1$ matrix $M=(a)$ is $\mathbb{A}$-clean iff $a \in \mathrm{U}(K)$.

(2) A coset of $\mathbb{A}_{n}(K)$ in $\mathbb{M}_{n}(K)$ consists of $\mathbb{A}$-clean matrices iff it has a coset representative from $\mathrm{GL}_{n}(K)$. Otherwise, it consists of non $\mathbb{A}$-clean matrices.

(3) The coset (modulo $\mathbb{A}_{n}(K)$ ) determined by a matrix $M$ contains a unique upper triangular matrix $M_{0}$ (obtained by adding the "obvious" alternating matrix to $M$ to kill all its entries below the diagonal). The matrix $M$ is alternating iff $M_{0}=0$.

(4) If $n$ is even, $\mathbb{A}_{n}(K)$ consists of $\mathbb{A}$-clean matrices. (Let $n=2 m$. If $H$ denotes the hyperbolic matrix $\left(\begin{array}{cc}0 & 1 \\ -1 & 0\end{array}\right)$, then $\operatorname{diag}(H, \ldots, H)$ (with $m$ copies of $H)$ is an invertible matrix in $\mathbb{A}_{n}(K)$.) If $n$ is odd, and $K \neq 0$ is commutative, $\mathbb{A}_{n}(K)$ consists of non $\mathbb{A}$-clean matrices, since every matrix in $\mathbb{A}_{n}(K)$ has a zero determinant: see, e.g. [La 2 : III.5.12].

The last remark above explained why in Theorem B the odd-size alternating matrices are "exceptions" (to $\mathbb{A}$-cleanness) in the field case. Next, we shall explain the exceptional cases in Theorem B in the case where $K$ is the field of two elements.

Proposition 2.3. Let $K$ be a division ring, and let $M \in \mathbb{M}_{2}(K)$. If $|K|>2, M$ is $\mathbb{A}$-clean. If $|K|=2, M$ is $\mathbb{A}$-clean iff it is not one of the six matrices in (1.1).

Proof. Our matrix $M=\left(\begin{array}{ll}a & b \\ c & d\end{array}\right)$ is $\mathbb{A}$-clean iff there exists $x \in K$ such that

$$
U:=M+\left(\begin{array}{cc}
0 & x \\
-x & 0
\end{array}\right)=\left(\begin{array}{cc}
a & b+x \\
c-x & d
\end{array}\right)
$$

is invertible. If $a d \neq 0$, choosing $x=c$ makes $U$ invertible. Now assume $a d=0$. If $|K|>2$, we can choose $x \in K \backslash\{c,-b\}$ to make $U$ invertible. However, if $|K|=2$ and $M$ is such that $a d=0$ and $b \neq c, x$ cannot be chosen to make $U$ invertible. The culprits are precisely the six matrices listed in (1.1).

The following lemma will provide the main technique for proving in the next section that certain matrices are $\mathbb{A}$-clean. 
Lemma 2.5. Let $K$ be any ring, and let $M$ be the block triangular matrix $\left(\begin{array}{cc}M_{1} & C \\ 0 & M_{2}\end{array}\right)$, where $M_{1} \in \mathbb{M}_{p}(K)$ and $M_{2} \in \mathbb{M}_{q}(K)$. If $M_{1}$ and $M_{2}$ are both $\mathbb{A}$-clean, then so is $M$.

Proof. For $i=1,2$, let $M_{i}=A_{i}+U_{i}$, where $A_{i}$ is alternating, and $U_{i}$ is invertible. From this, we get a decomposition

$$
M=\left(\begin{array}{cc}
A_{1} & 0 \\
0 & A_{2}
\end{array}\right)+\left(\begin{array}{cc}
U_{1} & C \\
0 & U_{2}
\end{array}\right) .
$$

On the RHS, the first matrix is alternating, and the second matrix is invertible (with inverse $\left(\begin{array}{cc}U_{1}^{-1} & -U_{1}^{-1} C U_{2}^{-1} \\ 0 & U_{2}^{-1}\end{array}\right)$ ). Thus, $M$ is $\mathbb{A}$-clean, as desired.

Remark 2.7. The converse of the Lemma is not true. For instance, the zero matrix $M \in \mathbb{M}_{2}(K)$ is $\mathbb{A}$-clean, but $M_{1}=M_{2}=(0)$ are not $\mathbb{A}$-clean (if $K \neq 0$ ).

If we want to prove that some matrix $M \in \mathbb{M}_{n}(K)$ (over a ring $K$ ) is $\mathbb{A}$-clean, we are always free to replace $M$ by an upper triangular matrix (by (2) and (3) in (2.2)). After this reduction, for any decomposition $n=p+q$ where $p, q \geq 1$, we can write $M$ in the block triangular form in Lemma 2.5. Thus, the job of proving the $\mathbb{A}$-cleanness of $M$ can be accomplished by doing the same for the "smaller" matrices $M_{i}$. In the following, we shall refer to this useful technique for proving $\mathbb{A}$-cleanness as the " $n=p+q$ " construction. This construction will be used over and over again in the proof of Theorem A and Theorem B below.

\section{Proof of Theorem A and Theorem B}

To prepare ourselves for the proof of Theorems A and B, we first prove the following result on $3 \times 3$ matrices over a division ring, leaving aside the field of two elements for the time being.

Proposition 3.1. Over a noncommutative division ring $K$, any matrix $M \in$ $\mathbb{M}_{3}(K)$ is $\mathbb{A}$-clean. Over a field $K$ with $|K|>2, M$ is $\mathbb{A}$-clean iff $M$ is not alternating.

Proof. As in (2.2)(3), we may replace $M$ by an upper triangular matrix $M_{0}=$ $\left(\begin{array}{ccc}u & a & b \\ 0 & v & c \\ 0 & 0 & w\end{array}\right)$. If one of the diagonal entries is nonzero, say $u \neq 0$, then the $" 3=$ $1+2$ " construction together with $(2.3)$ show that $M_{0}$ is $\mathbb{A}$-clean. Thus, we may focus on the case $u=v=w=0$. If $a, b, c$ are not all zero, we may assume, say, $a \neq 0$. Then

$$
M_{0}+\left(\begin{array}{ccc}
0 & 0 & 1 \\
0 & 0 & 1-c \\
-1 & c-1 & 0
\end{array}\right)=\left(\begin{array}{ccc}
0 & a & b+1 \\
0 & 0 & 1 \\
-1 & c-1 & 0
\end{array}\right) \in \mathrm{GL}_{3}(K)
$$

so $M_{0}$ is $\mathbb{A}$-clean. This (plus $(2.2)(3)$ ) completes the proof of $(3.1)$ when $K$ is a field with $|K|>2$. Now assume $a=b=c=0$; that is, $M_{0}=0$. If $K$ is a noncommutative division ring, $M_{0}$ does turn out to be $\mathbb{A}$-clean: we'll return to prove this in (4.6). 
Proof of Theorem A. Let $M \in \mathbb{M}_{n}(K)$, where $K$ is a noncommutative division ring. If $n=1$, clearly $M$ is $\mathbb{A}$-clean iff $M \neq(0)$. If $n>1$, we may assume, as before, that $M$ is upper triangular. If $n=2$ or 3 , we are done by (2.3) or (3.1). If $n \geq 4$, we can use the " $n=2+(n-2)$ " construction together with induction and (2.3) to show that $M$ is $\mathbb{A}$-clean.

Proof of Theorem B. Here $K$ is a field, and we take $M \in \mathbb{M}_{n}(K)$ to be upper triangular, with $M \neq 0$ in case $n$ is odd. (The latter corresponds to the nonalternating case for odd $n$.) We assume first that $|K|>2$. If $n$ is even, we can finish (as above) by the " $n=2+(n-2)$ " construction, using induction and (2.3). If $n$ is odd, we may write (after reindexing the rows and columns) $M=\left(\begin{array}{cc}M_{1} & C \\ 0 & M_{2}\end{array}\right)$, where $M_{1} \in \mathbb{M}_{3}(K)$ is nonzero and $M_{2} \in \mathbb{M}_{n-3}(K)$. Since $M_{1}$ is $\mathbb{A}$-clean by (3.1) and $n-3$ is even, this " $n=3+(n-3)$ " construction shows that $M$ is $\mathbb{A}$-clean.

We come now to the more tedious case $|K|=2$. If Theorem $\mathrm{B}$ is true for $n=3,4,5,6$ (as well as for $n=1,2$ ), the case $n \geq 7$ can be handled as follows. For even $n$, we can use the " $n=4+(n-4)$ " construction together with induction. For odd $n$, we can use the " $n=3+(n-3)$ " construction and the even case (after arranging that $M_{1} \neq 0$ in $\left.\mathbb{M}_{3}(K)\right)$.

Next, we handle the cases $n=5,6$, assuming the cases $n=3,4$.

(1) $n=6$. Here, we deal with $M=\left(\begin{array}{cc}M_{1} & C \\ 0 & M_{2}\end{array}\right)$ where $M_{i} \in \mathbb{M}_{3}(K)$ are upper triangular. We are done if $M_{1} \neq 0 \neq M_{2}$, so let us assume, say, $M_{1}=0$. In this case, the " $6=2+4$ " construction shows that $M$ is $\mathbb{A}$-clean.

(2) $n=5$. We may assume that all $m_{i i}=0$ (for otherwise the " $5=1+4$ " construction works). If $m_{i j}=1$ for all $i<j$, it is straightforward to check that

$$
M-\left(E_{15}-E_{51}\right) \in \mathrm{GL}_{5}(K) \text {, where } E_{i j} \text { are the matrix units. }
$$

Thus, we may assume, say, $m_{12}=0$, so now $M=\left(\begin{array}{cc}0 & C \\ 0 & M_{2}\end{array}\right)$ where $M_{2} \in \mathbb{M}_{3}(K)$ is upper triangular. If $M_{2} \neq 0$, this " $5=2+3$ " construction works. If, instead, $M_{2}=0$, then $C \neq 0$ (as $M \neq 0$ ). We may assume the first column of $C$ is nonzero. In this case, the " $5=3+2$ " construction shows that $M$ is $\mathbb{A}$-clean.

At this point, the two remaining cases below will complete the proof of Theorem B.

(3) $n=3$. If $m_{12}=m_{13}=m_{23}=0$, we have (say) $m_{11} \neq 0$, and the " $3=1+2$ " construction works. (Note that the $M_{2}$ in this construction is not one of the matrices in (1.1), so it is $\mathbb{A}$-clean.) We may therefore assume (say) $m_{12}=1$. Adding an alternating matrix $x\left(E_{13}+E_{31}\right)+y\left(E_{23}+E_{32}\right)$ to $M$, we get a matrix $U$ with

$$
\operatorname{det}(U)=m_{22} x^{2}+x(y+m)+(\text { terms not involving } x),
$$

where $m=m_{23}+m_{22} m_{13}$. If $m_{22}=0$, let $y=m+1$; we can then choose $x$ so that $\operatorname{det}(U)=1$. If $m_{22} \neq 0$, let $y=m$, and we can choose $x$ again to make $\operatorname{det}(U)=1$. (Note that $x^{2}=x$ for all $x \in K$.) Thus, $M$ is $\mathbb{A}$-clean.

(4) $n=4$. First assume some $m_{i i}$ is nonzero; say $m_{11}=1$. We may assume the lower right $3 \times 3$ corner of $M$ is zero, for otherwise the " $4=1+3$ " construction 
works. If some $m_{1 j}=0$, say $m_{12}=0$, the " $4=2+2$ " construction works since neither $M_{1}$ nor $M_{2}$ in this construction is among the matrices in (1.1). We may thus assume $M$ is the first matrix on the LHS of (3.4) below. In this case, adding the second (alternating) matrix on the LHS to $M$ yields the invertible matrix on the RHS:

$$
\left(\begin{array}{llll}
1 & 1 & 1 & 1 \\
0 & 0 & 0 & 0 \\
0 & 0 & 0 & 0 \\
0 & 0 & 0 & 0
\end{array}\right)+\left(\begin{array}{llll}
0 & 1 & 0 & 0 \\
1 & 0 & 0 & 1 \\
0 & 0 & 0 & 1 \\
0 & 1 & 1 & 0
\end{array}\right)=\left(\begin{array}{llll}
1 & 0 & 1 & 1 \\
1 & 0 & 0 & 1 \\
0 & 0 & 0 & 1 \\
0 & 1 & 1 & 0
\end{array}\right)
$$

In the remaining case, all $m_{i i}=0$. Since $M=0$ is no problem, we may assume, say, $m_{34}=1$. Adding to $M$ the alternating matrix

$$
x\left(E_{12}+E_{21}\right)+y\left(E_{23}+E_{32}\right)+\left(E_{14}+E_{41}\right) \in \mathbb{A}_{4}(K),
$$

we get a matrix $U$ with

$$
\operatorname{det}(U)=x\left(y+m_{23}\right)+(\text { terms not involving } x) .
$$

Taking $y=m_{23}+1$, we can choose $x$ such that $\operatorname{det}(U)=1$. Thus, in all cases, the non-alternating matrix $M$ is $\mathbb{A}$-clean.

\section{Alternating Matrices over Division Rings}

As we saw in $\S 3$, the determination of $\mathbb{A}$-clean matrices over a noncommutative division ring $K$ depends on the existence of a $3 \times 3$ alternating matrix in $\mathrm{GL}_{3}(K)$. In this section, we supply this existence result needed in the proof of Theorem A in $\S 3$, by investigating the invertibility of the matrix

$$
A=\left(\begin{array}{ccc}
0 & x & y \\
-x & 0 & z \\
-y & -z & 0
\end{array}\right) \in \mathbb{A}_{3}(K)
$$

Proceeding more generally, we have the following result over any ring $K$.

Proposition 4.2. Let $y \in \mathrm{U}(K)$ over a ring $K$. Then $A \in \mathrm{GL}_{3}(K)$ iff $w:=$ $x y^{-1} z-z y^{-1} x \in \mathrm{U}(K)$.

Proof. First assume $w \in \mathrm{U}(K)$. Let $E_{i j}(a)(i \neq j)$ denote the elementary matrix with $a$ in the $(i, j)$-th position. Right multiplying $A$ by $E_{32}\left(-y^{-1} x\right) E_{12}\left(-y^{-1} z\right)$, we get the matrix $B=\left(\begin{array}{ccc}0 & 0 & y \\ -x & w & z \\ -y & 0 & 0\end{array}\right)$. Since $y, w \in \mathrm{U}(K), B$ is invertible with

$$
B^{-1}=\left(\begin{array}{ccc}
0 & 0 & -y^{-1} \\
-w^{-1} z y^{-1} & w^{-1} & -w^{-1} x y^{-1} \\
y^{-1} & 0 & 0
\end{array}\right) .
$$

Therefore, $A$ is also invertible, with

$$
\begin{aligned}
A^{-1} & =E_{32}\left(-y^{-1} x\right) E_{12}\left(-y^{-1} z\right) B^{-1} \\
& =\left(\begin{array}{ccc}
y^{-1} z w^{-1} z y^{-1} & -y^{-1} z w^{-1} & y^{-1} z w^{-1} x y^{-1}-y^{-1} \\
-w^{-1} z y^{-1} & w^{-1} & -w^{-1} x y^{-1} \\
y^{-1} x w^{-1} z y^{-1}+y^{-1} & -y^{-1} x w^{-1} & y^{-1} x w^{-1} x y^{-1}
\end{array}\right) .
\end{aligned}
$$


Conversely, assume $A \in \mathrm{GL}_{3}(K)$. Then we also have

$$
B=A E_{32}\left(-y^{-1} x\right) E_{12}\left(-y^{-1} z\right) \in \mathrm{GL}_{3}(K) .
$$

From the form of the matrix $B$, it follows that $w \in \mathrm{U}(K)$, as desired.

In the case where $y$ is a central unit in $K$ and $w \in \mathrm{U}(K)$, the expression for $A^{-1}$ simplifies considerably. In fact, in this case, $w=y^{-1}(x z-z x)$. Thus, if $u:=(x z-z x)^{-1}$, we have $w^{-1}=u y$, and hence

$$
A^{-1}=\left(\begin{array}{ccc}
y^{-1} z u z & -z u & y^{-1}(z u x-1) \\
-u z & u y & -u x \\
y^{-1}(x u z+1) & -x u & y^{-1} x u x
\end{array}\right) .
$$

Remark 4.5. In Prop. 4.2, the criterion for the invertibility of $A$ was proved under the assumption that $y \in \mathrm{U}(K)$. This latter condition is, however, not a necessary condition for $A$ to be invertible. Here is an easy example. Let $K=\mathbb{M}_{2}(D)$, where $D$ is any nonzero ring, and choose

$$
x=E_{21}, \quad y=E_{12}, \quad \text { and } \quad z=E_{11}+E_{12}+E_{21}+E_{22}
$$

in $K$. If we identify $\mathbb{M}_{3}(K)$ with $\mathbb{M}_{6}(D), A$ becomes a $6 \times 6$ invertible matrix over $D$ (since it has determinant -1 over the center of $D$ ), and hence $A \in \mathrm{GL}_{3}(K)$. However, none of $x, y, z$ is a unit in $K$. In the case where $K$ is a division ring, no such example is possible, as will be clear from (4.6)(2) below.

The following consequence of Prop. 4.2 gives the result that was used in advance in the proof of Theorem $\mathrm{A}$ in $\S 3$.

Corollary 4.6. (1) If a ring $K$ has two elements $x, z$ such that $x z-z x \in \mathrm{U}(K)$, then for any $n>1$, there exists an invertible matrix $A_{n} \in \mathbb{A}_{n}(K)$, or equivalently, all matrices in $\mathbb{A}_{n}(K)$ are $\mathbb{A}$-clean. (For instance, $K$ can be any matrix ring $\mathbb{M}_{r}(D)$ with $r>1$, where $D$ is any ring.)

(2) Over a division ring $K$, the alternating matrix $A$ in (4.1) is invertible iff $y \neq 0$ and $x y^{-1} z \neq z y^{-1} x$. In this case, the Dieudonné determinant of $A$ is $y^{2}\left(x y^{-1} z-z y^{-1} x\right)$ in $K^{*} /\left[K^{*}, K^{*}\right]$. In particular, if $y \neq 0$ is central in $K$ and $x z \neq z x$, we have $A \in \mathrm{GL}_{3}(K)$.

Proof. (1) The existence of $A_{n}$ is automatic for $n$ even (by (2.2)(4)). For $n$ odd (and > 1), we can take $A_{n}$ to be $\operatorname{diag}(A, H, \ldots, H)$, where $H$ is the hyperbolic matrix, and $A$ is the matrix in (4.1) with the given $x, z$ and with $y=1$.

To see that $K=\mathbb{M}_{r}(D)$ (with $r>1$ ) is an example for (1), it suffices to check the cases $r=2,3$. For $r=2$, we can take $x=E_{12}, z=E_{21}$, for which

$$
x z-z x=E_{11}-E_{22} \in \mathrm{U}(K) .
$$

For $r=3$, we can take $x=E_{12}+E_{23}, z=E_{31}+E_{23}$, for which

$$
x z-z x=E_{13}+E_{21}-E_{32} \in \mathrm{U}(K) .
$$

(The fact noted here about $\mathbb{M}_{r}(D)$ will turn out to be crucial for the proof of a later result, Theorem $\mathrm{C}$ in $\S 5$.)

(2) Assume $K$ is a division ring. If $A \in \mathrm{GL}_{3}(K)$, we must have $y \neq 0$, for otherwise the first and third rows of $A$ become left linearly dependent. Thus, we have $y \in \mathrm{U}(K)$, and the "only if" part of (4.2) gives $x y^{-1} z \neq z y^{-1} x$. Conversely, 
if $y \neq 0$ and $x y^{-1} z \neq z y^{-1} x$, the "if" part of (4.2) implies $A \in \mathrm{GL}_{3}(K)$. In this case, in view of (4.3), the Dieudonné determinant of $A$ is the same as that of $B$. The latter determinant is, of course, $y^{2}\left(x y^{-1} z-z y^{-1} x\right)$; see $[\mathrm{Ar}]$.

Example 4.7. Let $F$ be a field, and $K$ be the division hull of the Weyl algebra $W$ generated over $F$ by $x, z$ with the relation $x z-z x=1$. Taking $y=1$, the matrix $A$ in (4.1) has Dieudonné determinant 1, and (4.4) gives

$$
A^{-1}=\left(\begin{array}{ccc}
0 & x & 1 \\
-x & 0 & z \\
-1 & -z & 0
\end{array}\right)^{-1}=\left(\begin{array}{ccc}
z^{2} & -z & z x-1 \\
-z & 1 & -x \\
x z+1 & -x & x^{2}
\end{array}\right) .
$$

This computation shows that the matrix $A$ is already invertible over the Weyl algebra $W$ itself (rather than just invertible over its division hull $K$ ).

Example 4.9. Let $K$ be a generalized quaternion algebra over a field $F$ of characteristic not 2. Say $K$ has generators $i, j, k$ with the relations $i^{2}=a$, $j^{2}=b$, and $k=i j=-j i$ (where $a, b \in F^{*}$ ). Then, for $x=i, y=j$ and $z=k$, we have $y \in \mathrm{U}(K)$ and

$$
w:=x y^{-1} z-z y^{-1} x=b^{-1}(i j k-k j i)=-2 a \in \mathrm{U}(F) \subseteq \mathrm{U}(K) .
$$

Thus, the alternating matrix $A$ in (4.1) is invertible, with

$$
A^{-1}=\left(\begin{array}{ccc}
0 & i & j \\
-i & 0 & k \\
-j & -k & 0
\end{array}\right)^{-1}=\frac{1}{2}\left(\begin{array}{ccc}
1 & -i / a & -j / b \\
i / a & -1 / a & k / a b \\
j / b & -k / a b & -1 / b
\end{array}\right),
$$

and (in case $K$ is a division algebra) $A$ has Dieudonné determinant $-2 a b$ in the factor group $K^{*} /\left[K^{*}, K^{*}\right]$. In the "classical" (Hamiltonian) case where $a=b=$ -1 , this inverse simplifies to

$$
A^{-1}=\frac{1}{2}\left(\begin{array}{ccc}
1 & i & j \\
-i & 1 & k \\
-j & -k & 1
\end{array}\right) .
$$

In this case, it is easy to check that $A^{2}+A=2 I$, so we could have obtained $A^{-1}$ directly as $(I+A) / 2$. In the case of generalized quaternion algebras, however, there is no such expression.

\section{The Case of Simple Artinian Rings}

In this section, we shall extend Theorems A-B further, from the case of division rings to that of simple artinian rings with (say) infinite centers. Throughout this section, therefore, $K$ denotes a simple artinian ring, which (according to Wedderburn's Theorem) can be taken to be of the form $\mathbb{M}_{r}(D)$, where $D$ is a division ring. Since we have already settled the case of division rings, there will be no loss of generality in assuming that $r>1$. On the surface, it might seem that the case of a simple artinian ring $K$ could be routinely reduced to the case of a division ring $D$, since any matrix ring $\mathbb{M}_{n}(K)$ is canonically isomorphic to $\mathbb{M}_{n r}(D)$. Unfortunately, under this isomorphism, although the invertible matrices correspond to one another, the alternating matrices do not! Therefore, a naive direct reduction of the problem to the division ring case is not possible. We shall, nevertheless, prove the 
following result, which extends Theorems A and B to simple artinian rings with "relatively large" (e.g. infinite) centers.

Theorem C. Let $D$ be a division ring with center $F$, and let $K$ be the simple artinian ring $\mathbb{M}_{r}(D)$ with $r>1$. Suppose that $|F|>3 r$. Then all matrices $M \in \mathbb{M}_{n}(K)$ with $n>1$ are $\mathbb{A}$-clean.

Here, there is no loss in leaving out the $n=1$ case in light of Remark (2.2)(1). The proof of this theorem is essentially based on standard techniques in linear algebra. However, these techniques must be invoked in the somewhat less familiar setting of linear algebra over division rings. The following fact about matrices over division rings is what we need as the main tool for proving Theorem $\mathrm{C}$.

Proposition 5.1. Let $N \in \mathbb{M}_{k}(D)$, where $D$ is a division ring with center $F$. Then there exists a polynomial $f(x) \in D[x]$ of degree $k$ such that, for any $a \in F$, $a I_{k}-N \in \mathrm{GL}_{k}(D)$ iff $f(a) \neq 0$.

(Note that, since $a$ is in the center of $D$, the evaluation of the polynomial $f(x)$ at $a$ is unambiguously defined.)

Proof. In the case where $D$ is a field, we would have $D=F$, so $f(x)$ could be taken as the characteristic polynomial $\operatorname{det}\left(x I_{k}-N\right)$ of the matrix $N$. For a division ring $D$, the above determinant no longer makes sense, so we have to come up with a reasonable substitute. The right tool to use here is the characteristic sequence for the linear transformation defined by $N$.

We think of $D^{k}$ as the left $D$-vector space of rows of length $k$. With $N$ fixed, we make $D^{k}$ into a left $D[x]$-module by letting $x$ act as the right multiplication of row vectors by the matrix $N$. Given this $D[x]$-action on $D^{k}$, we have the following short exact "characteristic sequence" of $D[x]$-modules:

$$
0 \longrightarrow D[x]^{k} \stackrel{\sigma}{\longrightarrow} D[x]^{k} \stackrel{\tau}{\longrightarrow} D^{k} \longrightarrow 0 .
$$

Here, $\sigma$ is the right multiplication (on row vectors) by the matrix $x I_{k}-N$, and $\tau$ is the unique $D[x]$-homomorphism from $D[x]^{k}\left(=D^{k}[x]\right)$ to $D^{k}$ that is the identity map on $D^{k}$. (For more details, see [Ba: p. 630], or [Laan: IV.3.2].)

Since $D[x]$ is a (left and right) Euclidean domain, we can reduce the matrix $x I_{k}-N \in \mathbb{M}_{k}(D[x])$ to a diagonal form $\operatorname{diag}\left(f_{1}, \ldots, f_{k}\right)$ by elementary row and column operations, where $f_{i} \in D[x]$. Therefore, we have (from (5.2)) a $D[x]$ module isomorphism

$$
D^{k} \cong \bigoplus_{i=1}^{k}\left(D[x] / D[x] f_{i}\right) .
$$

Taking $D$-dimensions, we have $k=\sum_{i=1}^{k} \operatorname{deg}\left(f_{i}\right)$. Let

$$
f(x):=f_{1}(x) \cdots f_{k}(x) \in D[x],
$$

which has degree $k$. (If $D=F$, this $f$ would have been the usual characteristic polynomial of the matrix $N$.) For any central element $a \in F$, consider the specialization ring homomorphism $D[x] \rightarrow D$ defined by $g(x) \mapsto g(a)$. This induces a specialization $\mathbb{M}_{k}(D[x]) \rightarrow \mathbb{M}_{k}(D)$, sending $x I_{k}-N$ to $a I_{k}-N$. Since $x I_{k}-N$ is equivalent to $\operatorname{diag}\left(f_{1}, \ldots, f_{k}\right), a I_{k}-N$ is equivalent to 
$\operatorname{diag}\left(f_{1}(a), \ldots, f_{k}(a)\right)$. From this, it follows immediately that $a I_{k}-N$ is invertible iff $\operatorname{diag}\left(f_{1}(a), \ldots, f_{k}(a)\right)$ is invertible; that is, iff

$$
f(a)=f_{1}(a) \cdots f_{k}(a) \neq 0 .
$$

Thus, $f(x) \in D[x]$ is the polynomial we sought.

We are now ready to prove the main result in this section.

Proof of Theorem C. For $K=\mathbb{M}_{r}(D)$ (with $r>1$ ), let $M \in \mathbb{M}_{n}(K)$ be our given matrix, where $n>1$. By (4.6)(1), there exists an invertible alternating matrix $A_{n} \in \mathbb{A}_{n}(K)$. Let $N:=-A_{n}^{-1} M \in \mathbb{M}_{n}(K)$ and identify $\mathbb{M}_{n}(K)$ with $\mathbb{M}_{n r}(D)$. Applying Proposition 5.1 with $k=n r$, choose a polynomial $f(x) \in D[x]$ (of degree $n r$ ) for $N$ as in Proposition 5.1. Since $f$ can have at most $n r$ distinct roots in the center $F$, we can choose $a \in F$ with $f(a) \neq 0$ provided that $|F|>n r$. Then $a I_{n r}-N=a I_{n r}+A_{n}^{-1} M$ will be invertible, and hence so is $U:=a A_{n}+M$. Since $-a A_{n} \in \mathbb{A}_{n}(K)$, it follows that $M=-a A_{n}+U$ is $\mathbb{A}$-clean in the matrix ring $\mathbb{M}_{n}(K)$, as desired. Thus, under the given assumption $|F|>3 r$, Theorem $\mathrm{C}$ holds for $n=2$ and $n=3$. But then the " $n=2+(n-2)$ " construction (plus induction) takes care of all of the remaining cases.

It seems likely that Theorem $\mathrm{C}$ would already hold without any assumption on the size of $F$. However, we have not been able to handle the cases where the center $F$ is too small.

\section{The Case of Semilocal Rings}

Recall that a (not necessarily commutative) ring $K$ is semilocal if the factor ring $K / \operatorname{rad}(K)$ is a semisimple ring [La $:$ p. 296]. (Here and in the following, $\operatorname{rad}(K)$ denotes the Jacobson radical of $K$.) In this section, we'll show that the work done in $\S \S 3-5$ is sufficient to give a complete determination of $\mathbb{A}$-clean matrices over "most" semilocal rings, including those $K$ for which $K / \operatorname{rad}(K)$ is a finite direct product of division rings (e.g. local rings and commutative semilocal rings). The key observation here is the following reduction fact on $\mathbb{A}$-cleanness modulo ideals in the Jacobson radical.

Proposition 6.1. Let $M \in \mathbb{M}_{n}(K)$ where $K$ is any ring, and let $J$ be an ideal of $K$ contained in $\operatorname{rad}(K)$. Then $M$ is $\mathbb{A}$-clean over $K$ iff its image $\bar{M} \in \mathbb{M}_{n}(K / J)$ is $\mathbb{A}$-clean over $K / J$.

Proof. The "only if" part is clear. For the converse, write $\bar{M}=\bar{A}+\bar{U}$, where $\bar{A} \in \mathbb{A}_{n}(K / J)$ and $\bar{U} \in \mathrm{GL}_{n}(K / J)$. Pick a matrix $A \in \mathbb{A}_{n}(K)$ lifting $\bar{A}$, and let $U:=M-A$. Then $U$ reduces to $\bar{U}$ modulo $J$, and the fact that $\bar{U}$ is invertible over $K / J$ implies that $U$ is invertible over $K$. Thus, $M=A+U$ is $\mathbb{A}$-clean over $K$.

In view of (6.1), the determination of $\mathbb{A}$-clean matrices over a semilocal ring $K$ is effectively reduced to the case where $K=\mathbb{M}_{r}(D)$ for a division ring $D$. In this case, our results in $\S \S 3-5$ were complete except in the case where $r>1$ and $D$ has a small center (in comparison to $r$ ). Barring the $r>1$ case, for instance, we have the following immediate consequence of (6.1) and Theorems A and B. 
Corollary 6.2. Let $K$ be a (semilocal) ring such that $K / \operatorname{rad}(K) \cong K_{1} \times \cdots \times K_{s}$, where the $K_{i}$ 's are division rings. For $M \in \mathbb{M}_{n}(K)$, let $M_{i}$ be the image of $M$ in $\mathbb{M}_{n}\left(K_{i}\right)$. Then $M$ is $\mathbb{A}$-clean iff each $M_{i}$ is $\mathbb{A}$-clean over $K_{i}$. Thus, if $n>1$, $M$ is not $\mathbb{A}$-clean iff, for some $i, K_{i}$ is a field and we have either

(1) $n$ is odd and $M_{i}$ is alternating, or

(2) $n=2,\left|K_{i}\right|=2$, and $M_{i}$ is one of the six matrices in (1.1).

In fact, the above conclusions apply already if $K / \operatorname{rad}(K)$ is an arbitrary direct product of division rings.

In the case where $K / \operatorname{rad}(K) \cong \mathbb{M}_{r}(D)$ where $r>1$ and $D$ is a division ring with an infinite center, it would follow from (6.1) and Theorem $\mathrm{C}$ that any matrix $M \in \mathbb{M}_{n}(K)$ with $n>1$ is $\mathbb{A}$-clean, etc.

\section{References}

[Al] A. A. Albert: Symmetric and alternate matrices in an arbitrary field. Trans. Amer. Math. Soc. 43 (1938), 386-436.

[Ar] E. Artin: Geometric Algebra. Interscience Publishers, New York, 1957.

[Ba] H. Bass: Algebraic K-Theory. Benjamin, Reading, Mass., 1968.

[CK] V. Camillo, D. Khurana, T. Y. Lam, W. K. Nicholson, and Y. Zhou: Continuous modules are clean. Journal of Algebra (to appear, 2006).

[La 1 T. Y. Lam: A First Course in Noncommutative Rings. Second edition, Graduate Texts in Math., Vol. 131, Springer-Verlag, Berlin-Heidelberg-New York, 2001.

[La2] T. Y. Lam: Serre's Problem on Projective Modules. Monographs in Mathematics, SpringerVerlag, Berlin-Heidelberg-New York, 2006.

[LS] T. Y. Lam and R. G. Swan: Syzygy modules, sectionable sequences, and matrices over commutative rings. In preparation.

[Ni] W. K. Nicholson: Lifting idempotents and exchange rings. Trans. Amer. Math. Soc. 229 (1977), 269-278.

[NVZ] W. K. Nicholson, K. Varadarajan, and Y. Zhou: Clean endomorphism rings. Arch. Math. (Basel) 83 (2004), 340-343.

[Se] M. Ó. Searcóid: Perturbation of linear operators by idempotents. Irish Math. Soc. Bull. 39 (1997), 10-13.

[Wo] K. G. Wolfson: An ideal-theoretic characterization of the ring of all linear transformations. Amer. J. Math. 75 (1953), 358-386.

[Ze] D. Zelinsky: Every linear transformation is a sum of two nonsingular ones. Proc. Amer. Math. Soc. 5 (1954), 627-630.

Department of Mathematics, University of California, Berkeley, California 94720

E-mail address: lam@math.berkeley.edu

Department of Mathematics, University of Chicago, Chicago, Illinois 60637

E-mail address: swan@math.uchicago.edu 